\title{
Error Analysis of Circular Gratings Angle-Measuring System
}

\author{
Shunqing Ren ${ }^{1, *}$, Qingbo Liu ${ }^{1}$ and Hongbo Zhao ${ }^{2}$ \\ ${ }^{1}$ Space Control and Inertial Technology Research Center, Harbin Institute of Technology, 150080 Harbin, China \\ ${ }^{2}$ Shanghai Institute of Satellite Engineering, 200240 Shanghai, China \\ *Corresponding author
}

\begin{abstract}
In order to analyze the error sources of circular gratings angle-measuring system, a series of coordinate systems were established on the gratings angle-measuring system through taking many mechanical errors into account, such as rotary errors of the axis system, installing eccentricity and tilt of circular gratings disc, graduation errors, readhead installation errors and etc. Then position and attitude relationships between two adjacent coordinate systems were established, thus the displacement error of gratings' graduation in the readhead coordinate system was calculated by homogeneous transformation method, so an angle-measuring error model was established, the relationship between the mechanical error sources and the angle-measuring errors under the condition of the different number of used readheads was expounded. According to the established error model and theoretical analysis, some conclusions were drawn, that is, adopting single readhead can not eliminate first harmonic errors resulted from the installation eccentricity error; using twin readheads not installed centrosymmetrically can not totally eliminate first harmonic errors, and some residual first order harmonic errors retained; applying four readheads can eliminate mechanical installation errors, but can not completely eliminate the graduation errors. Finally, an experiment was carried out on the circular gratings angle-measuring system mounted on an aerostatic bearings, some conclusions were verified, and it provided a theoretical basis for raising the angular position accuracy of the angle-measuring system.
\end{abstract}

Keywords-circular gratings; graduation errors; homogeneous transformation; angle-measuring system; rotary error

\section{INTRODUCTION}

Circular gratings angle-measuring system is more widely used in turntable because of its high accuracy, reliability, easier installation, etc. In the checking process of circular gratings angle-measuring system, it is discovered that the system using a single readhead contains a large first order harmonics errors, while using twin readheads, the angular errors of the system reduced significantly and the errors are more stable. In order to raise the accuracy of angle-measuring system, error sources and error mechanism must be researched and analyzed. Reference [1] analyzed the impact of the installing eccentricity on the gratings angle-measuring system. Reference $[2,3]$ introduced the circular gratings angle measurement module installed two readheads equal-spacedly, which has efficiently reduced the error introduced by scale eccentricity and distortion. Reference $[4,5]$ showed that the precision of circular grating system can be improved by using four readheads and digital addition technology. However, how do mechanical errors (rotary error, installing eccentricity and tilt of circular gratings disc, gratings' graduation error, readhead installation misalignment) influence the accuracy of the angle-measuring system? As far as the multiple readheads concerned, how do they eliminate all or part of these errors? This paper will derive the influence of these mechanical error sources on the accuracy of angle-measuring system by applying the homogeneous transformation method in propagating these error sources.

\section{ESTABLISHMENT OF HOMOGENEOUS TRANSFORMATION MATRIX}

As shown in Figure 1, the reference coordinate system $o_{0} x_{0} y_{0} z_{0}$ is fixed on the axis sleeve. Its origin $o_{0}$ is the intersection point of the circular gratings disc's middle cross section and the rotation axis line, and $o_{0} x_{0}$ orients to the center of readhead $\mathrm{A}$, $o_{0} Z_{0}$ is the rotation axis line, $o_{0} y_{0}$ is determined by the right-handed rule. Readhead $\lambda$ coordinate system $o_{\lambda} x_{\lambda} y_{\lambda} z_{\lambda}$, whose origin $o_{\lambda}$ coincides with $o_{1}$, and $o_{\lambda} x_{\lambda}$ orients to $\lambda$ readhead signed by angular position $\lambda$ which is around the counterclockwise direction of the $o_{0} z_{0}, o_{\lambda} z_{\lambda}$ is parallel to $o_{0} z_{0}$. The homogeneous transformation matrix (hereafter be shorted as $\mathrm{H}$ matrix ) of the reference coordinate system versus the $o_{\lambda} x_{\lambda} y_{\lambda} z_{\lambda}$ system is

$$
\boldsymbol{T}_{0}^{\lambda}=\operatorname{Rot}\left(z_{0},-\lambda\right)=\left[\begin{array}{cccc}
\cos \lambda & \sin \lambda & 0 & 0 \\
-\sin \lambda & \cos \lambda & 0 & 0 \\
0 & 0 & 1 & 0 \\
0 & 0 & 0 & 1
\end{array}\right]
$$




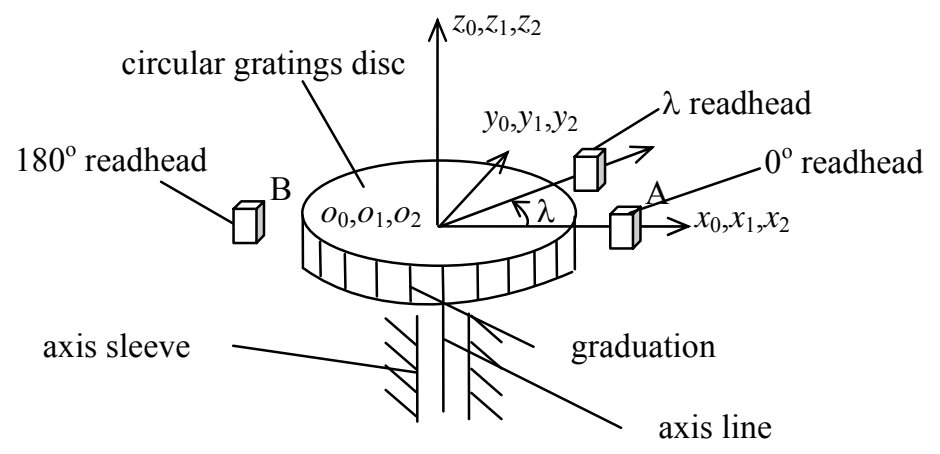

FIGURE I. SCHEMATIC DIAGRAM OF THE COORDINATE SYSTEMS

Axis coordinate system $o_{1} x_{1} y_{1} z_{1}$ is established by rotating angle $\alpha$ about the $o_{1} z_{1}$ counterclockwise, with the consideration of runout errors $\Delta x_{0}(\alpha), \Delta y_{0}(\alpha)$, the wobble errors
$\Delta \varphi_{x 0}(\alpha), \Delta \varphi_{y 0}(\alpha)$, axial slip error $\Delta z_{0}(\alpha)$ (when the axis is at angular position $\alpha$ ). So $\mathrm{H}$ matrix of the axis coordinate system versus the reference coordinate system is:

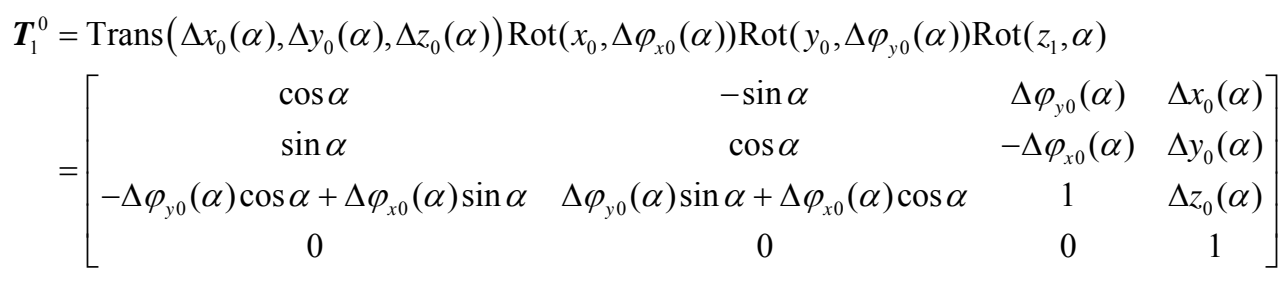

Circular gratings coordinate system $\mathrm{O}_{2} X_{2} y_{2} z_{2}$ is established with considering 2-D perpendicularity errors $\Delta \varphi_{x 1}, \Delta \varphi_{y 1}$ (also called installing tilts)of circular gratings disc plane versus rotary axis line $o_{0} z_{0}$, the installing eccentricity of gratings disc $\Delta L_{1}, \Delta L_{2}$, and height errors $\Delta L_{3}$ caused by circular gratings middle section not on the equal height of readhead. H matrix of circular gratings axis coordinate system versus the reference coordinate system is

$$
\begin{aligned}
\boldsymbol{T}_{2}^{1} & =\operatorname{Trans}\left(\Delta L_{1}, \Delta L_{2}, \Delta L_{3}\right) \operatorname{Rot}\left(x_{1}, \Delta \varphi_{x 1}\right) \operatorname{Rot}\left(y_{1}, \Delta \varphi_{y 1}\right) \\
& =\left[\begin{array}{cccc}
1 & 0 & \Delta \varphi_{y 1} & \Delta L_{1} \\
0 & 1 & -\Delta \varphi_{x 1} & \Delta L_{2} \\
-\Delta \varphi_{y 1} & \Delta \varphi_{x 1} & 1 & \Delta L_{3} \\
0 & 0 & 0 & 1
\end{array}\right]
\end{aligned}
$$

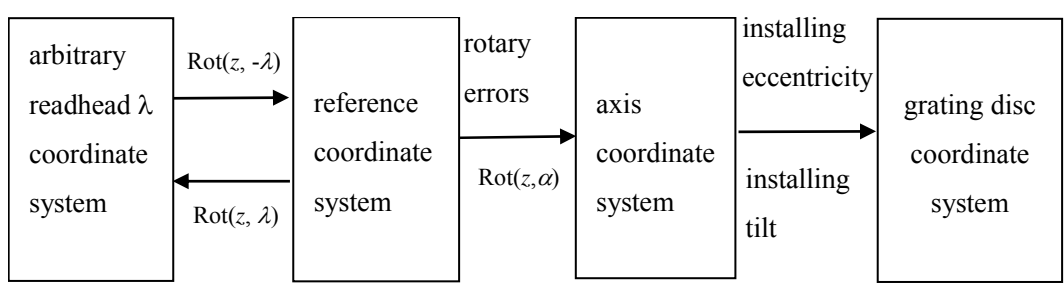

FIGURE II. THE POSITION AND ATTITUDE RELATIONSHIP AMONG COORDINATE SYSTEMS CONSIDERING MECHANICAL ERRORS

Figure 2 shows the position and attitude error propagating process through the established coordinate system. When the axis turns around $\alpha,-\alpha$ graduation of the circular gratings aligns with $0^{\circ}$ readhead, and $-\alpha+\lambda$ graduation aligns with $\lambda$ position readhead. In the gratings disc coordinate system, the coordinate of angle $\beta$ graduation is $\left[\begin{array}{lllll}R \cos \beta & R \sin \beta & 0 & 1\end{array}\right]^{\mathrm{T}}, R$ is the radius of the circle determined by all graduations. When the axis rotates angle $\alpha,-\alpha+\lambda$ graduation's homogeneous coordinate errors in the $y$ direction will be studied emphatically, because this error divided by the radius $R$ is none other than the angular

$$
\Delta y(\lambda)=R \sin (\alpha+\beta-\lambda)+\Delta L_{1} \sin (\alpha-\lambda)+\Delta L_{2} \cos (\alpha-\lambda)-\Delta x_{0}(\alpha) \sin \lambda+\Delta y_{0}(\alpha) \cos \lambda
$$


$\Delta y(\lambda)$ denotes displacement error of gratings' $(\lambda-\alpha)$ graduation in the $y$ direction observed by $\lambda$ readhead. When $\lambda=0$, $\Delta y(0)$ represents displacement error of $-\alpha$ graduation in the $y$ direction which is reflected in $0^{\circ}$ readhead. $\Delta y_{0}(\pi)$ is the displacement error of $-\alpha+\pi$ graduation reflected by $180^{\circ}$ readhead. In the same manner, when the installing position error of $180^{\circ}$ readhead is $\Delta \theta$, the displacement error of $\pi+\Delta \theta-\alpha$ graduation observed by the $180^{\circ}$ readhead is $\Delta y(\pi+\Delta \theta)$.

\section{ERROR MECHANISM ANALYSIS}

Based on the Figure 1 and the above analysis, the main reason for giving rise to the angular error of the circular gratings is the displacement error $\Delta y(\lambda)$. Comparative analysis will be carried out aiming at the different number of readheads, including a single readhead, twin readheads and four readheads, their main error sources and the advantages of twin or four readheads over the single readhead will be analyzed.

\section{A. Single readhead}

If there is only one readhead $\mathrm{A}$, that is $\lambda=0$, and the error of gratings' graduation is $\Delta \alpha(-\alpha)$, which is a function of angular position $\alpha$. The corresponding gratings' graduation is $\beta=-\alpha+\Delta \alpha(-\alpha)$. With Eq. 4, the error of the circular gratings angle-measuring system is:

$$
\Delta \alpha(\alpha)=\Delta \alpha_{0}+\Delta \alpha_{1 s} \sin \alpha+\Delta \alpha_{1 c} \cos \alpha+\Delta \alpha_{2 s} \sin 2 \alpha+\Delta \alpha_{2 c} \cos 2 \alpha+\ldots \ldots
$$

Substituting it into Eq.3, so the angle-measuring error is

$$
\Delta \varphi=\Delta \alpha_{0}+\Delta \alpha_{2 c} \cos 2 \alpha-\Delta \alpha_{2 s} \sin 2 \alpha+\Delta \alpha_{4 c} \cos 4 \alpha-\Delta \alpha_{4 s} \sin 4 \alpha+\ldots \ldots
$$

By Eq. 10, the eccentricity errors $\Delta L_{1}, \Delta L_{2}$, and the runout error $\Delta y_{0}(\alpha)$ can be compensated by twin readheads. Part of the errors of gratings' graduation can be compensated, namely odd order harmonic errors of gratings' graduation can be automatically compensated, while even order harmonic errors can not be eliminated. Thus the main error source of the angle-measuring system using twin readheads which are perfectly installed centrosymmetrically is even order harmonic errors in graduation errors.

$$
\begin{aligned}
\Delta \varphi(\pi+\Delta \theta) & =\frac{\Delta y(\pi+\Delta \theta)}{R} \approx \Delta \alpha(-\alpha+\Delta \theta+\pi)+\frac{\Delta L_{1}}{R} \sin (\alpha-\pi-\Delta \theta)+\frac{\Delta L_{2}}{R} \cos (\alpha-\pi-\Delta \theta) \\
& -\frac{\Delta x_{0}(\alpha)}{R} \sin (\Delta \theta+\pi)+\frac{\Delta y_{0}(\alpha)}{R} \cos (\Delta \theta+\pi) \\
& \approx \Delta \alpha(-\alpha+\Delta \theta+\pi)-\frac{\Delta L_{1}}{R} \sin \alpha+\frac{\Delta L_{1} \Delta \theta}{R} \cos \alpha-\frac{\Delta L_{2}}{R} \cos \alpha-\frac{\Delta L_{2} \Delta \theta}{R} \sin \alpha+\frac{\Delta x_{0}(\alpha) \Delta \theta}{R}-\frac{\Delta y_{0}(\alpha)}{R}
\end{aligned}
$$

If the installed error is $\Delta \theta=2^{\circ}$, gratings radius is $R=100 \mathrm{~mm}$, the eccentricity error is $\Delta L_{1}=0.02 \mathrm{~mm}$. Then the error term $\frac{\Delta \theta \Delta L_{1}}{R}=$

$\frac{\Delta}{R}=1.44^{\prime \prime}$,this error can't be ignored, and
$-\frac{\Delta L_{1} \Delta \theta}{R} \cos \alpha+\frac{\Delta L_{2} \Delta \theta}{R} \sin \alpha$ is regarded as residual first order harmonic errors affected by the common influence of eccentricity $\Delta L_{1}, \Delta L_{2}$ and the installing error $\Delta \theta$. If $\Delta L_{1}, \Delta L_{2}$ are

\section{Twin readheads with installing errors}

If angle-measuring system has two readheads, and readhead $\mathrm{A}$ is installed at $0^{\circ}$, where readhead $\mathrm{B}$ is installed at $\pi+\Delta \theta$, relative installing error of $\mathrm{B}$ versus $\mathrm{A}$ is $\Delta \theta$. Then the corresponding graduation errors is $\Delta \alpha(\pi+\Delta \theta-\alpha)$, as for readhead $\mathrm{B}$ : $\beta=-\alpha+\pi+\Delta \theta+\Delta \alpha(-\alpha+\pi+\Delta \theta)$, the error of angle-measuring system only using readhead $B$ is

Calculating the average of these two readheads A and B, the

The graduation error $\Delta \alpha$ is a periodic function versus the 8) controlled within $0.01 \mathrm{~mm}$, and $\Delta \theta$ within $0.1^{\circ}$, so the error term $\frac{\Delta \theta \Delta L_{1}}{R}=0.036^{\prime \prime}$, the influence of residual first order harmonic error on the angle-measuring error can be ignored.

Averaging readouts of twin readheads, so 


$$
\begin{aligned}
\Delta \varphi & =\frac{\Delta y(0)+\Delta y(\pi+\Delta \theta)}{2 R} \\
& \approx \Delta \alpha_{0}+\frac{\Delta L_{1} \Delta \theta}{2 R} \cos \alpha-\frac{\Delta L_{2} \Delta \theta}{2 R} \sin \alpha-\Delta \alpha_{2 s} \sin 2 \alpha+\Delta \alpha_{2 c} \cos 2 \alpha-\Delta \alpha_{4 s} \sin 4 \alpha+\Delta \alpha_{4 c} \cos 4 \alpha+\ldots \ldots
\end{aligned}
$$

From the Eq. 12, the main error sources of the angle-measuring system with twin readheads which are not installed centrosymmetrically contain part of graduation errors and residual first order harmonic error. To speak more accurately, odd harmonics in graduation errors can be automatically compensated, while the even harmonics can not be eliminated, and the residual first harmonic can not be ignored. The main error sources are the even order harmonics in errors of graduation, the residual first order harmonic errors which are produced by the installing error $\Delta \theta$ of twin readheads and the installing eccentricity $\Delta L_{1}, \Delta L_{2}$ of the gratings disc.

\section{Four readheads}

Installing four readheads A, B, C, D as shown Figure 3, assuming that the installing error is less than $0.1^{\circ}$, and the installing eccentricity of circular gratings disc is less than $0.01 \mathrm{~mm}$, the residual first order harmonic error is negligible, according to the above methods the average value of four readheads is:

$$
\begin{aligned}
\Delta \varphi & =\frac{\Delta y(0)+\Delta y\left(\frac{\pi}{2}\right)+\Delta y(\pi)+\Delta y\left(\frac{3 \pi}{2}\right)}{4 R} \\
& \approx \Delta \alpha_{0}-\Delta \alpha_{45} \sin 4 \alpha+\Delta \alpha_{4 c} \cos 4 \alpha-\Delta \alpha_{85} \sin 8 \alpha+\Delta \alpha_{8 c} \cos 8 \alpha+\ldots . . .
\end{aligned}
$$

From the Eq. 13, when the installing errors of 4 readheads and the eccentricity of grating disc are controlled within a certain range, the main error sources are $4^{\text {th }}$ order, $8^{\text {th }}$ order, $12^{\text {th }}$ order harmonic errors in the graduation errors. So angle-measuring system using 4 readheads will attain higher accuracy.

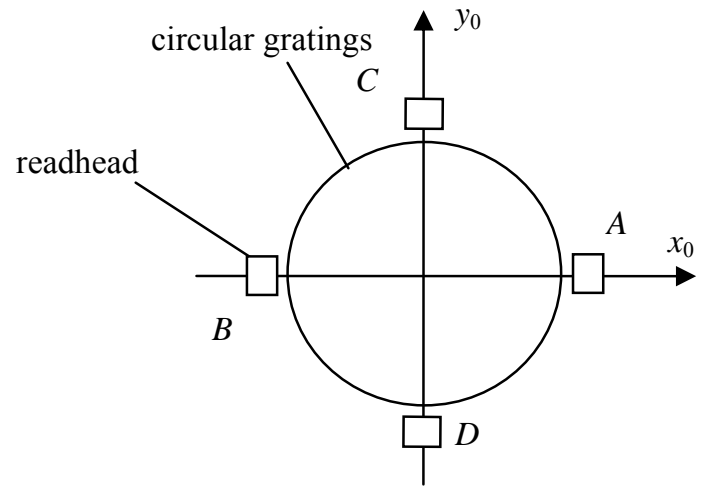

FIGURE III. FOUR-READHEAD CIRCULAR GRATINGS ANGULAR-MEASURING SYSTEM

\section{EXPERIMENT RESULTS}

Error checking experiment was carried out on circular gratings angle-measuring system which was installed on the high-precision air bearing. When only one readhead was adopted, the eccentricity of the gratings disc was adjusted $80 \mu \mathrm{m}$ deliberately, and the measured angular position errors $\Delta \varphi_{1 i}$ $(i=0,1,2, \ldots, 23)$ were shown in Tab. 1 , Fourier analysis versus $\Delta \varphi_{1 i}$ was conducted, and first order harmonic error was:

$$
-31.1 " \cos \alpha+80.7 " \sin \alpha
$$

Two coefficients can be calculated as follows.

$$
\frac{2}{24} \sum_{i=0}^{23}\left(\Delta \varphi_{1 i} \cos \frac{2 \pi i}{24}\right)=-31.1, \frac{2}{24} \sum_{i=0}^{23}\left(\Delta \varphi_{1 i} \sin \frac{2 \pi i}{24}\right)=80.7
$$

Then twin readheads $\mathrm{A}$ and $\mathrm{B}$ were used, but installing eccentricity error was also $80 \mu \mathrm{m}$. The measured data $\Delta \varphi_{2 i}$ was shown in Tab. 1, Fourier analysis for $\Delta \varphi_{2 i}$ was also conducted as mentioned above, the first order harmonic error was:

$$
-0.75^{\prime \prime} \cos \alpha-1.35^{\prime \prime} \sin \alpha
$$

Adjusting the eccentricity error within $10 \mu \mathrm{m}$ by way of a dial test indicator and a rubber mallet, and adjusting the installing error of readhead B as small as possible, the residual first order harmonics error was greatly decreased, the measured errors were shown as $\Delta \varphi_{3 i}$ in Tab. 1 , the residual first order harmonic error was:

$$
-0.16^{\prime \prime} \cos \alpha+0.34 " \sin \alpha
$$

From the first order harmonic analysis for the measured errors, this showed that the first order harmonics errors had to do with the number of the readheads, the magnitudes of installing errors about the gratings disc and the readheads. So the installing errors of the readhead and the eccentricity of the circular gratings disc must be adjusted as small as possible. 
TABLE I. ANGULAR POSITION ERRORS IN THREE SITUATIONS

\begin{tabular}{c|c|c|c}
\hline$\alpha_{i}\left({ }^{\circ}\right)$ & $\Delta \varphi_{1 i}\left({ }^{\prime \prime}\right)$ & $\Delta \varphi_{2 i}\left({ }^{\prime \prime}\right)$ & $\Delta \varphi_{3 i}\left({ }^{\prime \prime}\right)$ \\
\hline 0 & 0.0 & 0.0 & 0.00 \\
\hline 15 & 21.6 & -0.4 & 0.00 \\
\hline 30 & 44.2 & -0.6 & 0.23 \\
\hline 45 & 66.0 & -0.8 & 0.19 \\
\hline 60 & 85.4 & -0.9 & 0.38 \\
\hline 75 & 101.1 & -1.2 & 0.31 \\
\hline 90 & 112.2 & -0.8 & 0.41 \\
\hline 105 & 117.5 & -0.6 & 0.47 \\
\hline 120 & 116.9 & -0.1 & 0.50 \\
\hline 135 & 110.4 & 0.1 & 0.47 \\
\hline 150 & 98.8 & 0.8 & 0.48 \\
\hline 165 & 82.0 & 1.2 & 0.50 \\
\hline 180 & 62.5 & 1.4 & 0.35 \\
\hline 195 & 40.1 & 1.9 & 0.28 \\
\hline 210 & 17.4 & 2.1 & 0.19 \\
\hline 225 & -4.2 & 2.0 & -0.16 \\
\hline 240 & -23.1 & 2.2 & -0.07 \\
\hline 255 & -39.0 & 1.9 & -0.29 \\
\hline 270 & -49.3 & 1.9 & -0.26 \\
\hline 285 & -54.4 & 1.7 & -0.28 \\
\hline 300 & -54.1 & 1.3 & -0.26 \\
\hline 315 & -47.6 & 1.1 & -0.19 \\
\hline 330 & -35.8 & 0.7 & -0.13 \\
\hline 345 & -19.5 & 0.6 & 0.10 \\
\hline & & &
\end{tabular}

\section{CONCLUSIONS}

(1)Mechanical error analysis is conducted on the circular gratings angle-measuring system, the impacts of the error sources on the accuracy of the angle-measuring system was derived. For a single readhead, the main error sources are graduation errors, the installing eccentricity of the gratings disc and runout errors of the rotation axis system.

(2)For the twin readheads installed centrosymmetrically, the eccentricity error and rotary error can be automatically compensated. Only odd order harmonic errors in the graduation errors can be eliminated, so the main error sources are the even order harmonic errors in the graduation errors.

(3)If the twin readheads are not installed accurately centrosymmetrically, namely installing error of two readheads exists. The residual first order harmonic error is produced by common influence of the eccentricity of gratings disc and installing error of twin readheads. So the magnitudes of installing errors must be adjusted as small as possible.

(4) For the four-readhead system, when the installing error is within $0.1^{\circ}$ and the eccentricity error of the disc is less than $0.01 \mathrm{~mm}$, the main error sources is $4^{\text {th }}, 8^{\text {th }}, 12^{\text {th }} \ldots$ order harmonics in errors of graduation, so the system will attain higher accuracy.

\section{REFERENCES}

[1] Yang-kuan Guo, Yu-he Li, Qing-xiang Li, Juan Gong, Zhang-wei Chen,
Error compensation for eccentric motions of circular gratings, J. Journal of Tsinghua University (Sci\&Tech). 45 (2005) 178-181.

[2] Song Tang, Ming Fang, Lei Yao, Error Compensation for Installation Eccentric of Circular Gratings, J. Metrology \& Measurement Technique. 6 (2013) 11-12.

[3] Min Wu, Research on Inertia Testing Turntable's Circular Grating Angle Module and Friction Compensation, D. Harbin Institute of Technology, Harbin, 2011.

[4] Dong-feng Su, Study of Key Techniques on High Precision Calibration Turntable Grating Angle Measuring System, D. Changchun Institute of Optics, Fine Mechanics and Physics, 2011.

[5] Dong-feng Su, Zhi-jun Xu, Ji-qiang Jia, Bo Liu, Da-wei Li, Read-head design for improving the precision of circula grating angular measuring system, J. Journal of Electronic Measurement and Instrument. 27 (2013) 653-657.

[6] Xi-jun Chen, Zhen-huan Wang, Qing-shuang Zeng, Angle measurement error and compensation for decentration rotation of circular gratings, J. Journal of Harbin Institute of Technology (new series). 17 (2010) 536-539.

[7] G.X. Zhang, C.H. Wang, Z. Li, Improving the accuracy of angle measurement system with optical gratings, J. CIRP Annals Manufacturing Technology. 43 (1994) 457-460.

[8] Lian-Dong Yu, Yang Ding, Wen-Tao Cheng, Compensation of angle measurement error of circular gratings for parallel dual-joint coordinate measuring machine, J. Journal of Nanjing University of Science and Technology. 33 (2009) 659-662.

[9] Guan-bin Gao, Wen Wang, Keng Lin, Zi-chen Chen, Error compensation and parameter identification of circular grating angle sensors, J. Optics and Precision Engineering. 18 (2010) 1766-1772.

[10] Ren-jie Zhao, Wen-li Ma, Improving the accuracy of new-type encoders using error harmonic compensation, J. Electronic Instrumentation Customer. 3 (2009) 69-71. 